\title{
DISPONIBILIDAD A PAGAR POR LA EFICIENCIA ENERGÉTICA DE LAS VIVIENDAS; UN CASO DE ESTUDIO EN BARCELONA
}

\section{WILLINGNESS TO PAY FOR THE ENERGY EFFICIENCY OF DWELLINGS; A CASE STUDY IN BARCELONA}

\section{Carlos Marmolejo Duarte (1)}

Profesor Titular de Universidad

Av. Diagonal 649, 4⿳亠丷厂 Planta. 08028. Barcelona España

E-mail: carlos.marmolejo@upc.edu

Teléfono: +34 934054385

\section{Rolando Biere Arenas (1)}

Asistente de Investigación (PQS)

Av. Diagonal 649, 4ª Planta. 08028. Barcelona España

E-mail: rolando.biere@upc.edu

Teléfono: +34 934016396

\section{Francesc Daumal Domenech (2)}

Catedrático de Universidad

Av. Diagonal 649, 7a Planta. 08028. Barcelona España

E-mail: francesc.daumal@upc.edu

Teléfono: +34 934010867

\section{Jocelyne de Botton Halfon (2)}

Profesora Colaboradora Doctora

Av. Diagonal 649, $7^{\underline{a}}$ Planta. 08028. Barcelona España

E-mail: ioyce.debotton@upc.edu

Teléfono: +34 934054458

\section{Carlos Pérez Lamas ${ }^{(1)}$}

Profesor Titular de Escuela Universitaria (TEU)

Av. Diagonal 649, 4를 Planta. 08028. Barcelona España

E-mail: carlos.perez@upc.edu

Teléfono: +34 934016398

(1) Universidad Politécnica de Cataluña - Barcelona Tech (UPC), Departamento de Tecnología de la Arquitectura (TA) ETSAB, Centro de Política de Suelo y Valoraciones (CPSV)

(2) Universidad Politécnica de Cataluña - Barcelona Tech (UPC), Departamento de Tecnología de la Arquitectura (TA) ETSAB, Grup de recerca FORM+

Palabras Clave: Ahorro energético, encuestas, Certificados de Eficiencia Energética, Valoración Contingente

Key words: Energy saving, surveys, Energy Performance Certificates, Contingent Valuation

\section{Resumen}

Este trabajo se desarrolla en el marco del proyecto EnerValor ¿Cuánto nos importa la calificación energética? Un análisis del nivel de comprensión de los EPC, confianza percibida e impacto sobre las preferencias y valores residenciales (BIA2015-63606-R - MINECO/FEDER),

Citación: MARMOLEJO, C. et al. Disponibilidad a pagar por la eficiencia energética de las viviendas; un caso de estudio en Barcelona. En: Libro de proceedings, CTV 2018. XII Congreso Internacional Ciudad y Territorio Virtual. "Ciudades y Territorios Inteligentes". UNCuyo, Mendoza, 5-7 septiembre 2018. Barcelona: CPSV, 2018, p. 339-356. 
cuyo investigador principal es el primer autor, en el que los demás coautores son miembros del equipo de investigación y trabajo.

En el Estado Español, la trasposición de la Energy Performance of Buildings Directive (EPBD) 2002/91/CE (refundida en la Directiva 2010/31/UE), se ha producido mediante la promulgación de diversos Reales Decretos: el RD 314/2006, que determina los requisitos de eficiencia energética; el RD 1027/2007, que fija los requisitos de inspección de calderas y climatización y; los RD 47/2007 y 235/2013 que determinan, respectivamente, los procedimientos de certificación de edificios nuevos y existentes. Entre otras cosas, esta política promueve la construcción de edificios nuevos y la rehabilitación energética de los existentes a través de la universalización de los certificados energéticos en la práctica totalidad de las transacciones inmobiliarias.

El objetivo principal de este trabajo es determinar cuánto se modifica la Disponibilidad a Pagar $(D A P)$ por la eficiencia energética, si se informa a los usuarios de formas diferentes acerca de sus repercusiones económicas y ambientales, explicándoles además lo que realmente miden los Certificados de Eficiencia Energética.

La hipótesis inicial es que, si se informa más claramente a la demanda acerca de las características e implicancias de la certificación energética, se genera una mayor DAP por parte de los usuarios.

El estudio se realiza en Barcelona y, para alcanzar el objetivo anterior y contrastar la hipótesis mencionada se acude al método de Valoración Contingente (VC) para obtener la disposición a pagar (DAP) por parte de los usuarios, mediante la aplicación de una encuesta con tres variantes.

1) Uso de unidades técnicas para informar sobre el consumo energético y las emisiones, sin explicar lo que miden los EPC,

2) Uso de unidades ilustrativas para informar sobre las repercusiones económicas y ambientales, sin explicar lo que miden los EPC, y

3) Uso de unidades ilustrativas para informar sobre las repercusiones económicas y ambientales, explicando además lo que miden los EPC.

Una vez obtenidos los resultados, se realizarán los análisis para determinar si existen diferencias estadísticamente significativas (Test de ANOVA de las medias, etc.) y, además, determinar los factores que explican la DAP en las tres variantes de la encuesta.

\section{Abstract}

The development of this paper has been within the framework of the project EnerValue. Does energy qualification on housing really matter? An analysis on EPCs comprehension, perceived confidence and impact on householder's preferences and residential values (BIA2015-63606-R - MINECO/FEDER), whose principal researcher is the first author, in which the other co-authors are members of the research team.

In Spain, the transposition of the Energy Performance of Buildings Directive (EPBD) 2002/91/CE (consolidated in the Directive 2010/31/EU), has been produced through the enactment of several Royal Decrees. RD 314/2006, which determines the energy efficiency

Citación: MARMOLEJO, C. et al. Disponibilidad a pagar por la eficiencia energética de las viviendas; un caso de estudio en Barcelona. En: Libro de proceedings, CTV 2018. XII Congreso Internacional Ciudad y Territorio Virtual. "Ciudades y Territorios Inteligentes". UNCuyo, Mendoza, 5-7 septiembre 2018. Barcelona: CPSV, 2018, p. 339-356. 
requirements; RD 1027/2007, which sets the boiler and air conditioning inspection requirements and both; RD 47/2007 and 235/2013 that determine, respectively, the certification procedures of new and existing buildings. In this context, housing rehabilitation processes have been initiated, energy certification of them (many with poor procedures) and some energy rehabilitation of buildings in socially degraded neighborhoods.

The main objective of this work is to determine how much the Willingness to Pay (WTP) by energy efficiency is modified, if users are informed in different ways about the economic and environmental repercussions of its, explaining, in addition, what Energy Performance Certificates (EPC) actually measure.

The initial hypothesis is that if the demand receives more clear information about the characteristics and implications of the energy certification, the users generate a greater WTP.

The study is carried out in Barcelona and the Contingent Valuation (CV) method has been be used to obtain the Willingness to Pay (WTP) by users, through the application of a survey with three variants.

1) Use of technical units to report about energy consumption and emissions, without explaining what the EPC measures,

2) Use of illustrative units to report about economic and environmental impacts, without explaining what the EPC measures, and

3) Use of illustrative units to inform about the economic and environmental repercussions, explaining in addition those that measure the EPC.

Once obtained the results, the analyze has been carried out to determine if there are statistically significant differences (ANOVA Test of the means, etc.) and, in addition, to determine the factors that explain the WTP in the three variants of the survey.

\section{Antecedentes}

Actualmente hay suficiente evidencia científica que permite corroborar que el calentamiento global del sistema climático es inequívoco y que presenta diversas manifestaciones, como por ejemplo "Ios aumentos observados del promedio mundial de la temperatura del aire y del océano, el deshielo generalizado de nieves y hielos y el aumento del promedio mundial del nivel del mar" (IPCC 2007). Tampoco se discute la preponderancia del origen antropogénico de estos cambios (Trenberth et al., 2007), existiendo múltiples pruebas de ello. Por ejemplo, las emisiones mundiales de GHG por efecto del hombre, han aumentado en un 70\% entre 1970 y 2004. También existe un elevado consenso acerca de que las ciudades tienen un protagonismo especial en este contexto de cambio climático. Éstas son responsables del $75 \%$ del consumo global de energía y del $80 \%$ de las emisiones gases efecto invernadero (GHG), según el Centre for Human Settlements (ONU-HABITAT, 2007). También hay claras evidencias sobre los altos costes de la producción y distribución de la energía, así como de la dependencia de las importaciones energéticas y sus posibles repercusiones geopolíticas (CE, 2008) que han posicionado a la eficiencia energética como uno de los aspectos relevantes de la actualidad, tanto así que la Unión Europea (UE) la ha definido como un área de actuación estratégica. 
Es muy relevante que, en el viejo continente, los edificios sean responsables de más del $40 \%$ del consumo energético, que el $63 \%$ de éste corresponda al uso residencial (Poel et al., 2007) y el $36 \%$ a las emisiones de CO2 (WWF, 2010). Todos estos aspectos ponen de manifiesto que el sector de la construcción, es clave para cumplir con los objetivos 20-20-20, establecidos por el protocolo de Kyoto (EPBD, 2010). En este contexto general, en 2002, la Comisión Europea aprobó la Energy Performance of Buildings Directive (EPBD) 2002/91/CE, refundida en la Directiva 2010/31/UE que (si se implementa integralmente) prevé una reducción del $6,5 \%$ de la energía final equivalente demandada en la UE (De Ayala et al., 2016). Una de las principales finalidades de la Directiva es dar transparencia a la situación de la eficiencia energética de los edificios, mediante la creación de los Energy Performance Certificates (EPC) y su consiguiente etiqueta de calificación energética de los edificios y las viviendas. Estas etiquetas permiten establecer una diferenciación inmobiliaria cuyo objetivo final es la promoción y rehabilitación del parque de edificios existentes, no eficientes en materia energética, para mejorar sus condiciones en este sentido. Por ello, la directiva exige a los estados su transposición a sus legislaciones propias, con el objetivo de determinar requisito y sistemas de cálculo específicos y adecuados a la realidad climática, energética y constructiva de cada uno (García-Hooghuis y Neila, 2013).

Como ya se ha indicado, la trasposición de la EPBD en el Estado Español, se ha producido mediante la promulgación de diversos Reales Decretos: el RD 314/2006, que determina los requisitos de eficiencia energética; el RD 1027/2007, que fija los requisitos de inspección de calderas y climatización y; los RD 47/2007 y RD 235/2013 que determinan, respectivamente, los procedimientos de certificación de edificios nuevos y existentes. Entre otras cuestiones específicas, esta política promueve la construcción de edificios nuevos y la rehabilitación energética de los existentes, a través de la universalización de los certificados de eficiencia energética (EPC) prácticamente en la totalidad de las transacciones inmobiliarias. Específicamente el RD 235/2013 obliga, desde el 1 de junio de 2013, a que los inmuebles que se vendan o alquilen, dispongan de un EPC y que su publicidad (a efectos comerciales) contenga informe de su clasificación energética. Sin embargo, a pesar de que han transcurrido cinco años, desde esta obligatoriedad en todo el territorio español, en general, su aplicación en lo referente a la información de la etiqueta energética es muy limitada (Checa y Biere, 2017).

La relación entre las etiquetas verdes, que precedieron a los EPC (BREAM, HQE, Minergie, Leed, Green Mark, Energy Star, etc.) y los precios de alquiler y venta de los inmuebles, está ampliamente estudiada. Por ejemplo, por Eicholtz, Kok \& Quigley (2010) para oficinas LEED y Energy Star o Fuerst \& McAllister (2011) para el mercado de oficinas inglés, que demostraron una relación de proporcionalidad inversa entre el rendimiento y la calificación energética del esquema BREEAM. Sin embargo, a pesar de los más de quince años trascurridos desde la publicación de la EPBD, no son muchos los estudios que han analizado la relación entre la clasificación energética introducida por esta directiva y la formación de los precios inmobiliarios. Mudgal, Lyons y Cohen (2013) realizaron una revisión sistemática de los primeros estudios sobre el impacto de los EPC en el mercado residencial europeo y actualizaciones de éstos se encuentran en Fuerst et al. (2015) y en Ramos et al. (2015).

A fin de obtener evidencias empíricas de la efectividad de la política de diferenciación inmobiliaria introducida por la EPBD y del impacto de los EPC sobre el mercado residencial, existen dos estudios pioneros realizados en Holanda. El trabajo de Brounen \& Kok (2011) analizó la incidencia de las nuevas etiquetas verdes en los precios residenciales y sus resultados determinaron una correlación positiva entre las viviendas mejor calificadas y los precios de venta de las transacciones. Este trabajo asume que las calificaciones energéticas

Citación: MARMOLEJO, C. et al. Disponibilidad a pagar por la eficiencia energética de las viviendas; un caso de estudio en Barcelona. En: Libro de proceedings, CTV 2018. XII Congreso Internacional Ciudad y Territorio Virtual. "Ciudades y Territorios Inteligentes". UNCuyo, Mendoza, 5-7 septiembre 2018. Barcelona: CPSV, 2018, p. 339-356. 
son una medida categórica de la eficiencia de las viviendas, considerando como base comparativa la calificación "D" (intermedia). Se descubre que el precio marginal varía entre un $10 \%$ en la etiqueta "A" y un $-5 \%$ en la etiqueta "G". Es decir, se generan, respectivamente, primas o penalizaciones de mercado, si se está encima o por debajo de la situación de referencia. Por su parte Kok \& Jennen (2012) estudiaron la incidencia de los EPC en el mercado europeo, encontrando que las oficinas con la calificación "C" (en relación con la calificación "D") tenían un valor superior al $4.7 \%$ en sus precios de transacción de alquiler. El trabajo del Hyland et al. (2013), realizado en diferentes ciudades irlandesas, fue el primero en comparar simultáneamente la incidencia de los EPC en el mercado de alquiler y venta, a partir de precios de oferta de ambos mercados. En general, este trabajo determinó que la incidencia de la etiqueta energética es mayor en el mercado de venta que en el de alquiler. Por ejemplo, una vivienda calificada como "A" (en relación con otra calificada con "D", con todo lo demás igual) tiene una prima de mercado del $9,30 \%$ en el caso de la venta, mientras que, si se trata del mercado de alquiler, la prima sólo es del 1,80\%.

Por su parte, en el contexto español, los estudios iniciales realizados, se han limitado a evaluar la influencia de las certificaciones en los precios. De Ayala, Galarraga y Spadaro (2016) utilizan valores de venta declarados por una muestra de encuestados de 5 ciudades (Madrid, Bilbao, Sevilla, Vitoria y Málaga) y mediante un cálculo propio de la clase energética, determinan que las viviendas, cuya clasificación se sitúa en "A", "B" o "C" tienen un valor, en opinión de sus propietarios, superior en un 9,8\% que aquellas situadas en "D", "E", "F" o "G". Por su parte, Marmolejo (2016) utiliza valores de oferta de viviendas en venta en Barcelona y determina que hay un sobreprecio de 5,11\% por pasar de la clase "G" a la "A", o del 9,62\%, aceptando que las personas perciben la clase energética como una variable categórica. Por su parte Checa y Biere (2017), mediante una encuesta aplicada a 150 personas, en zonas específicas de los barrios del Ensanche, Gracia y Sant Andreu, de Barcelona, determinaron que existe un gran desconocimiento de la población, acerca de las implicaciones de los EPC y que se requiere más tiempo, desde su aplicación, para una mejor comprensión de su conveniencia y necesidad, así como para su exigencia generalizada por parte de los usuarios. En esta misma línea, en una primera implementación de una encuesta similar a la que se utiliza en este trabajo, aplicada a 260 encuestados también en Barcelona, Marmolejo, García-Hooghuis y García-Masiá (2017) determinaron que detrás de la disposición a pagar, no solo hay factores financieros, como el nivel de renta o los costes energéticos evitados, sino también sociales y, además, que el nivel de conocimiento de los EPC y la sobrevaloración del régimen en propiedad frente al alquiler, también inciden en las decisiones de pago, sugiriendo la integración de la política energética en el contexto más amplio de la política de vivienda. Sin embargo, a pesar de estos trabajos previos, es necesario seguir investigando acerca del real nivel de comprensión de los EPC, por parte de los hogares españoles y sus implicaciones sobre la política energética de los edificios y las viviendas.

\section{Hipótesis y objetivos}

\subsection{Hipótesis}

En este contexto indicado, la hipótesis inicial de este trabajo es que; si se informa más claramente a la demanda, acerca de las características e implicaciones de la certificación energética, se genera una mayor DAP por parte de los usuarios.

Citación: MARMOLEJO, C. et al. Disponibilidad a pagar por la eficiencia energética de las viviendas; un caso de estudio en Barcelona. En: Libro de proceedings, CTV 2018. XII Congreso Internacional Ciudad y Territorio Virtual. "Ciudades y Territorios Inteligentes". UNCuyo, Mendoza, 5-7 septiembre 2018. Barcelona: CPSV, 2018, p. 339-356. 


\subsection{Objetivos}

El principal objetivo que se persigue, es determinar cuánto se modifica la Disponibilidad a Pagar $(D A P)^{1}$ por la eficiencia energética de una vivienda, si se informa a los usuarios de formas diferentes acerca de las repercusiones económicas y ambientales de ésta y si, además, se les explica lo que realmente miden los Certificados de Eficiencia Energética.

Consecuentemente con éste, los objetivos específicos son: a) Evaluar el nivel de conocimiento de los EPC por parte de los hogares encuestados; b) Determinar si existe disposición a pagar por viviendas más eficientes energéticamente, y en caso favorable, $c$ ) Determinar las razones subyacentes, en esta disposición a pagar (DAP).

\section{Metodología}

Si bien, desde el 1 de junio de 2013, en todo el territorio español es obligatorio contar con la etiqueta energética, desde el momento de la publicitación de un inmueble en oferta, para favorecer la transparencia y la información en esta materia y facilitar las decisiones de compra y alquiler informadas, según datos de Marmolejo (2016), únicamente un 12\% de las ofertas plurifamiliares en Barcelona ${ }^{2}$ incluye esta información. En el mismo estudio se señala además que, de las ofertas con información energética, las clases entre " $A$ " y "C" representan solo un $6,65 \%$. Esto pone de relieve que, existen muy pocas viviendas adaptadas a los requerimientos del DB-HE del Código Técnico de la Edificación (CTE).

En consideración de este aspecto anterior y para solucionar el problema de escasez de transacciones de viviendas de clases energéticas altas y, sobretodo, alcanzar el objetivo del trabajo y poder contrastar la hipótesis, se utiliza el método de Valoración Contingente (VC), para obtener la disposición a pagar (DAP) por parte de los usuarios. Como señala Azqueta (1996), el tener una medida de valor para los bienes sin mercado o posibles beneficios, es útil para la toma de decisiones con respecto a los posibles cambios, inversión en la conservación y mejora de un bien, ..., etc. La VC permite evaluar estos posibles cambios futuros o beneficios no disponibles aún en el mercado, definiendo un mercado hipotético que incluye los cambios potenciales (Mitchell y Carson, 1989) para que los encuestados indiquen su DAP. Parece importante recordar, que la Valoración Contingente (VC) como método para analizar un bien sin mercado (en este caso los escenarios de disposición a pagar en función de la información detallada a la demanda y de las características e implicancias de la certificación energética) ya ha sido utilizada y validada previamente por diversos autores, inicialmente respecto de bienes medioambientales; midiendo pérdidas o ganancias de bienestar de los encuestados ante el cambio ofrecido (Riera, 1994); valoración de uso de espacios naturales (Del saz y Suarez, 1998; Sánchez Sáez y Segado, 2013) o beneficios de mejoras constructivas, como los aislamientos acústicos en viviendas (Romo et al., 2013), entre otros.

El cambio evaluado en este trabajo es la mejora en la clase energética de una vivienda convencional (en un edificio) entre medianeras de 3 habitaciones, 2 baños, con una superficie de $81,5 \mathrm{~m}^{2}$ útiles, ubicado en Barcelona. Éste responde a la tipología dominante de apartamentos de reciente construcción, según un análisis multivariante construido a partir de

\footnotetext{
1 Se debe recordar que este concepto, desarrollado desde la microeconomía, se utiliza para expresar la cantidad máxima que una persona estaría dispuesta a pagar por adquirir o usar un bien o un servicio determinado.

2 Utilizando los datos de uno de los principales portales inmobiliarios con ofertas de esta ciudad.
} 
4.000 observaciones por Centro de Política de Suelo y Valoraciones. En concreto, la mejora energética ofrecida representa pasar de la clase "E" a la clase "A", la máxima contemplada por la transposición española. (Marmolejo et al., 2017)

\subsection{La encuesta aplicada}

Para la obtención de los datos y la construcción de la base de análisis, se aplica una encuesta, en Barcelona, a una muestra total de 750 encuestados, con tres variantes (250 encuestados en cada una), cuyas particularidades se detallan a continuación:

1) Uso de unidades técnicas para informar sobre el consumo energético y las emisiones, sin explicar lo que miden los EPC,

2) Uso de unidades ilustrativas para informar sobre las repercusiones económicas y ambientales, sin explicar lo que miden los EPC, y

3) Uso de unidades ilustrativas para informar sobre las repercusiones económicas y ambientales, explicando además lo que miden los EPC.

La encuesta informa que se está realizando una investigación sobre preferencias residenciales y que debe ser contestada (siempre) por una persona mayor de edad, que participe en la toma de decisiones sobre la vivienda. Es importante indicar que las tres variantes de la encuesta se estructuran en tres grandes apartados: a) contextualización, para determinar el conocimiento previo de los EPC y los hábitos energéticos por parte de los usuarios encuestados; b) valoración contingente, donde se detalla el cambio ofrecido por pasar de la clase "E" a la "A" (sus repercusiones económicas y ambientales, manteniendo igual todos los demás atributos arquitectónicos y locativos) y c) perfil socio-residencial, del encuestado y de su vivienda.

Los aspectos básicos de diferenciación, considerados en cada una de las tres variantes de la encuesta aplicada se presentan, a continuación, en la Tabla 1.

\section{Tabla 1. Aspectos básicos de las tres variantes de encuesta}

\begin{tabular}{|c|c|c|c|}
\hline Variante & $\begin{array}{c}\text { Unidades de } \\
\text { consumo energético }\end{array}$ & Unidades de emisiones & $\begin{array}{c}\text { Información sobre lo } \\
\text { que miden los EPC }\end{array}$ \\
\hline EVP1 & $\mathrm{kW} / \mathrm{h} / \mathrm{m} 2 / \mathrm{año}$ & $\mathrm{CO} / \mathrm{m} 2 / \mathrm{año}$ & $\mathrm{NO}$ \\
\hline EVP2 & Euros/mes & $\begin{array}{c}\text { Árboles necesarios para } \\
\text { absorber el CO2 } \\
\text { Equivalente en } \mathrm{km} \\
\text { recorrido por un coche }\end{array}$ & $\mathrm{NO}$ \\
\hline EVP3 & Euros/mes & $\mathrm{Sí}$ \\
\hline
\end{tabular}

Siguiendo una metodología validada, para informar las repercusiones en la economía familiar y el medio ambiente (que generarían las mejoras planteadas), los encuestados son informados tanto del ahorro en la factura energética, como de las potenciales emisiones de CO2. Como se aprecia en la tabla anterior, las unidades de medida fueron planteadas, para que sean fáciles de comprender por los encuestados. En cuanto al ahorro de consumo en la factura eléctrica, se

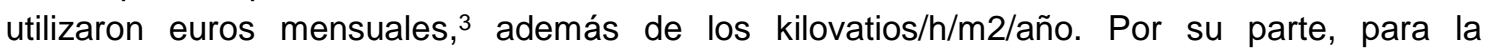

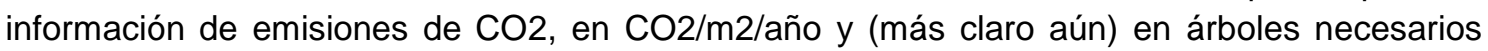
para absorber el $\mathrm{CO} 2$ y en el equivalente en kilómetros que recorre un automóvil.

\footnotetext{
${ }^{3}$ Unidad temporal que coincide con la periodicidad de los ingresos y los gastos del grueso de la población.
} 
Para facilitar la comprensión de la encuesta aplicada, a continuación, se explican algunas de las alternativas/diferencias de sus tres variantes. En la Figura 1 se muestran las opciones de una de las preguntas para determinar el nivel de conocimiento de los EPC. En ésta, en las variantes EVP1 y EVP2 sólo se pregunta al encuestado, su opinión acerca de los aspectos que se consideran para determinar la calificación energética, mientras que en la variante EVP3, además, se le entrega información respecto de lo que implican los tramos de eficiencia energética, tanto en ahorro en las facturas de luz y gas, como en emisiones de $\mathrm{CO} 2$.

Figura 1. Nivel de conocimiento de los Certificados de Eficiencia Energética
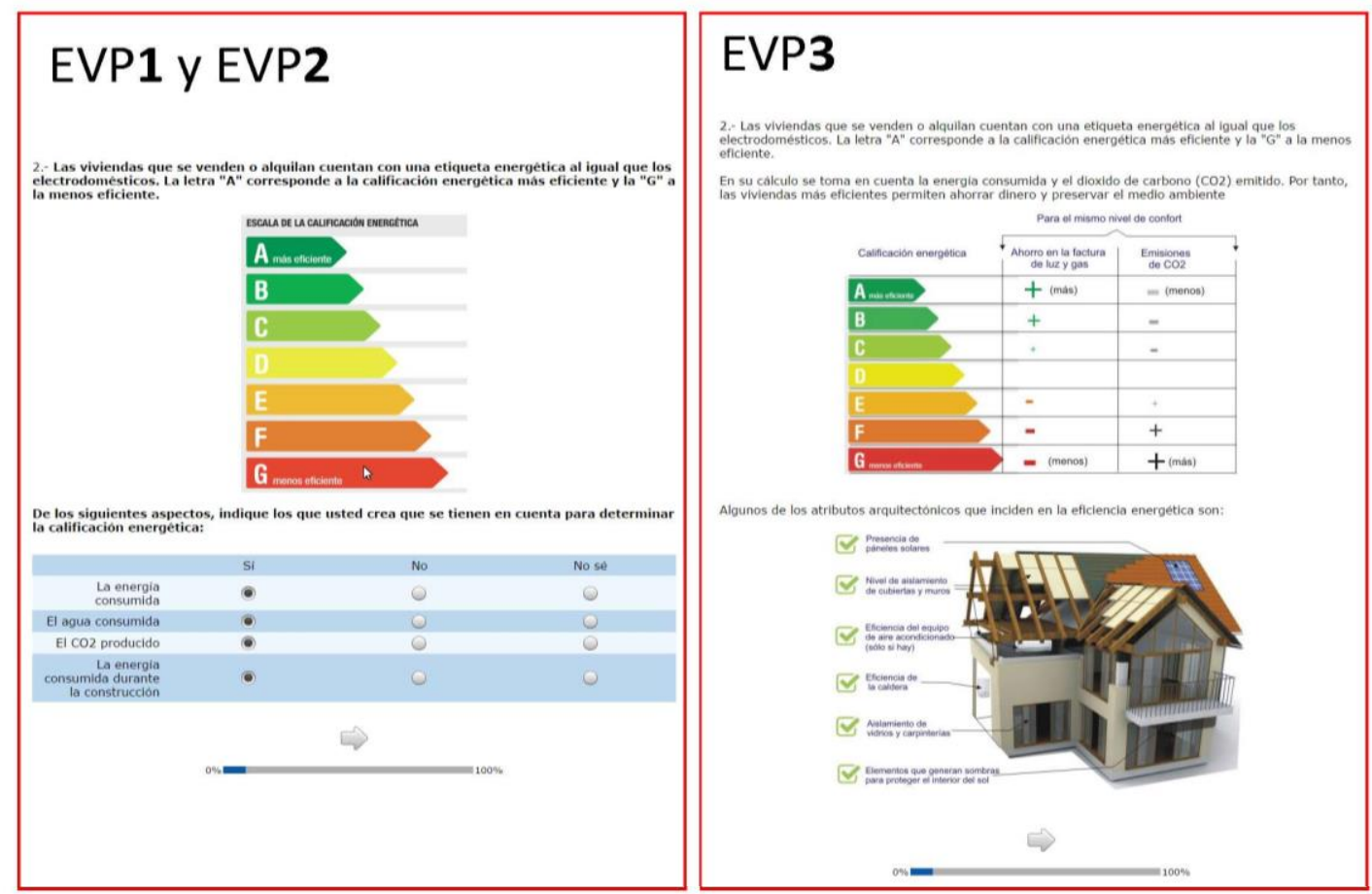

Fuente: Elaboración propia.

Posteriormente, en las tres variantes se presentan las características posibles de un piso de 3 habitaciones, con todas sus características iguales (zonas comunes, habitaciones adicionales, climatización interna, calidad de los acabados, calificación energética y coste en $€ /$ mes del pago por alquiler o hipoteca). Sin embargo, en lo referente a las opciones de calificación energética, si bien se mantienen las características, se modifica su presentación. Una vez que se conocen las características tipo de las viviendas, se presentan alternativas de elección (hasta cuatro veces) en base a paquetes de características y precios $(€ /$ mes) diferenciados. También se pide al encuestado que indique su preferencia por compra o alquiler. En lo que se refiere estrictamente a la disposición a pagar, se presenta al encuestado lo que implicaría el cambio por pasar de una calificación energética " $E$ " a una "A", la más eficiente energéticamente. Considerando que el ahorro energético y la reducción de $\mathrm{CO} 2$ son resultado de las estimaciones (para la misma tipología) realizada por García-Navarro et al. (2014) y Barboza (2016). Se decide: a) actualizar el coste energético a fecha actual y trasladarlo a Barcelona, en base a la diferencia de coste de energía; 2) realizar un modelo de regresión para estimar la media del consumo energético, el coste de la factura energética y las emisiones; y 3 ) convertir las unidades de emisiones en $\mathrm{km}$ recorridos equivalentes. ${ }^{4}$

\footnotetext{
${ }^{4}$ Se utilizan datos del Instituto para la Diversificación Energética, sobre emisiones de CO2 de coches nuevos.
}

Citación: MARMOLEJO, C. et al. Disponibilidad a pagar por la eficiencia energética de las viviendas; un caso de estudio en Barcelona. En: Libro de proceedings, CTV 2018. XII Congreso Internacional Ciudad y Territorio Virtual. "Ciudades y Territorios Inteligentes". UNCuyo, Mendoza, 5-7 septiembre 2018. Barcelona: CPSV, 2018, p. 339-356. 
Una vez aplicados estos criterios, las diferencias se presentan, en la EVP1, mediante un ahorro en las facturas de $900 \mathrm{~kW} / \mathrm{h} / \mathrm{año}$ y una reducción de CO2 de $2.300 \mathrm{~kg} / \mathrm{año}$ y, en las EVP2 y EVP3, mediante un ahorro en las facturas de $30 € /$ mes y una reducción de $\mathrm{CO} 2$ equivalente a $16.000 \mathrm{~km}$ recorridos por un turismo. Ante las opciones las opciones resultantes, se pregunta directamente si estaría dispuesto a pagar un poco más por el alquiler o hipoteca al mes. ${ }^{5} \mathrm{Si}$ bien se plantea una respuesta abierta, se utiliza un valor referencia del ahorro potencial en la factura energética, según las siguientes opciones: a) Sí, más de $30 €$; b) Sí, menos de $30 €$ y c) No pagaría nada, para el caso de plantearse comprar o alquilar el piso calificado con " $A$ ". En ambos casos favorables, para posteriormente poder analizar con más precisión la DAP, como segundo paso, se pide concretar una cifra, en base a sus posibilidades económicas.

Luego se pretende entender, tanto para las respuestas favorables como para las desfavorables, las razones de la DAP o de su negativa. Para ello se plantean opciones con las posibles razones subyacentes en la decisión. Esta información permite conocer, en el caso de una DAP positiva, si dicho sobrepago deriva de ahorros potenciales, de una conciencia en la preservación del ambiente/la salud pública o, incluso, de una eventual recompensa psicológica derivada de un consumo responsable. (Marmolejo et al., 2017)

Las preguntas finales son para conocer las características tanto de la vivienda actual, como de evaluación de las actividades del encuestado, para conocer su sensibilidad en materia de sostenibilidad, nivel de estudios, sexo, edad, ocupación, nivel de ingresos del hogar, etc.

Una vez obtenidos los resultados, se realizan los análisis para determinar si existen diferencias estadísticamente significativas (aplicando el Test de ANOVA de las medias, etc.) y, además, para determinar los factores que explican la DAP en las tres variantes de la encuesta.

\section{Análisis y resultados}

En este apartado se presentan los resultados de los diversos análisis realizados, en relación a los tres objetivos específicos del trabajo: a) Evaluar el nivel de conocimiento de los EPC por parte de los hogares encuestados; b) Determinar si existe disposición a pagar por viviendas más eficientes energéticamente, y c) Determinar las razones subyacentes, en esta disposición a pagar (DAP) o de su rechazo.

\subsection{Conocimiento de los EPC}

Como ya han demostrado algunos estudios previos, el desconocimiento de los EPC y de las implicaciones de tener una u otra calificación energética, ha sido uno de los grandes escollos a la hora de que los usuarios (compradores o arrendatarios potenciales) exijan conocer la etiqueta energética en la publicación del bien inmueble ofertado. Tanto así que la gran mayoría de estos indican en su oferta calificación energética "en trámite". Por ello ha parecido importante que el conjunto de los encuestados indicara que elementos creían que se consideraban para determinar la clase energética residencial, segmentados en: energía consumida, agua consumida, emisiones de $\mathrm{CO} 2$ y energía consumida durante la construcción.

\footnotetext{
5 Se utiliza el mes, como unidad conocida por la población. Asimismo, una DAP expresada en términos mensuales tiene relación con el ahorro ofrecido en la factura energética que también es mensual (o bimensual). Y todo ello tiene relación con el hecho de que los ingresos familiares y, por ende, los presupuestos suelen ser mensuales. 
Tabla 2. Nivel de conocimiento de los EPC

\begin{tabular}{|c|c|c|c|c|c|c|c|c|}
\hline \multirow[t]{2}{*}{ Conocimiento EPC } & \multirow[t]{2}{*}{ Toda la muestra } & \multicolumn{3}{|c|}{ Máximo nivel de estudios } & \multicolumn{4}{|c|}{ Edad } \\
\hline & & Pregrado & Grado & Postgrado & 18 a 30 & 31 a 45 & 46 a 65 & Más de 65 \\
\hline Nulo & $1 \%$ & $2 \%$ & $1 \%$ & $0 \%$ & $1 \%$ & $0 \% \rrbracket$ & $2 \%$ & $0 \%$ \\
\hline Bajo & $6 \%$ & $10 \%$ & $7 \%$ & $8 \%$ & $9 \%$ & $4 \%$ & $7 \%$ & $25 \%$ \\
\hline Medio_bajo & $30 \%$ & $54 \%$ & $43 \%$ & $42 \%$ & $46 \%$ & $41 \%$ & $48 \%$ & $38 \%$ \\
\hline Medio_alto & $20 \%$ & $22 \%$ & $34 \%$ & $32 \%$ & $32 \%$ & $31 \%$ & $26 \%$ & $25 \%$ \\
\hline Alto & $10 \%$ & $13 \%$ & $15 \%$ & $18 \%$ & $11 \%$ & $24 \%$ & $17 \%$ & $13 \%$ \\
\hline Aciertos & 1,67 & 2,34 & 2,55 & 2,59 & 2,45 & 2,75 & 2,50 & 2,25 \\
\hline \multirow[t]{3}{*}{ Sig. de ANOVA } & & & 0,015 & & & & 0,242 & \\
\hline & & \multicolumn{3}{|c|}{ Ingresos } & & & & \\
\hline & & Medios bajos & Medios altos & altos & & & & \\
\hline $\begin{array}{l}\text { Nulo } \\
\end{array}$ & & $1 \%$ & $0 \%$ & $1 \%$ & & & & \\
\hline Bajo & & $13 \%$ & $7 \%$ & $4 \%$ & & & & \\
\hline Medio_bajo & & $50 \%$ & $44 \%$ & $42 \%$ & & & & \\
\hline Medio_alto & & $26 \%$ & $31 \%$ & $35 \%$ & & & & \\
\hline Alto & & $10 \%$ & $18 \%$ & $18 \%$ & & & & \\
\hline Aciertos & & 2,3 & 2,59 & 2,65 & & & & \\
\hline Sig. de ANOVA & & & 0,024 & & & & & \\
\hline
\end{tabular}

Fuente: elaboración propia. Nota: La segmentación por ingresos responde a los terciles, siendo los límites 2.400 y 3.600 euros netos/hogar/mes. En relación al nivel de estudios se considera como Pregrado: primaria, secundaria y bachillerato, como Grado: diplomatura y licenciatura y como Postgrado: máster y doctorado.

La Tabla 2 muestra que sólo un $10 \%$ de los encuestados tiene un conocimiento alto de lo que miden los EPC, mientras que el mayor porcentaje, un 30\% tiene conocimientos medios bajos. Si se analiza la muestra, segmentada según sus características socio-demográficas, se puede destacar lo siguiente: a) A mayor nivel de formación e ingresos, mayor es el nivel de conocimiento de los EPC. Los encuestados que tienen "estudios de postgrado" y los de "ingresos altos" presentan respectivamente aciertos de 2,59 y 2,65 y b) A mayor edad hay un menor el nivel de conocimiento, de hecho, el segmento de "más de 65 años" solo tuvo un acierto de 2,25, medio punto por debajo del 2,75 obtenido por los encuestados del rango "entre 31 y 45 años". Como consecuencia de esto, se hace evidente que los grupos de mayor edad, así como los de menor formación y los de menor nivel de ingresos, deberían ser informados acerca del significado y repercusiones de los EPC, sobretodo porque muy probablemente, sus viviendas se verían altamente beneficiados por el hecho de introducir mejoras en eficiencia energética, a diferencia de los grupos socioeconómicos más favorecidos, cuyas viviendas suelen tener mejores condiciones energéticas.

Aunque la diferencia de clasificación entre las viviendas en escalones ordinales "G" - "A" permite comparar de manera sencilla los inmuebles, una de las cuestiones más cuestionadas en estudios previos (Lainé, 2011) son las unidades técnicas que utilizan los EPC para indicar las repercusiones económicas y ambientales de las edificaciones. Los $\mathrm{kWh} / \mathrm{m} 2$ y las toneladas de CO2 dificultan su comprensión por parte de la ciudadanía. La Tabla 3 muestra claramente que la inmensa mayoría de las personas, un $75 \%$ prefiere que el consumo sea expresado en euros y solo una cuarta parte, en las unidades técnicas previstas. Un $57 \%$ de la muestra prefiere que las emisiones de contaminación se expresen en el equivalente de árboles necesarios para absorber el $\mathrm{CO} 2$ y un $15 \%$ en kilómetros de recorrido equivalente de un turismo (sumando ambas un $72 \%$ ), mientras que sólo un $28 \%$ prefiere que sean expresadas en toneladas de $\mathrm{CO} 2$. Llama la atención la similitud entre estos dos resultados, que permiten indicar que prácticamente las tres cuartas partes de los encuestados prefieren las unidades descriptivas a las unidades técnicas definidas por la legislación, poco claras para la población.

Citación: MARMOLEJO, C. et al. Disponibilidad a pagar por la eficiencia energética de las viviendas; un caso de estudio en Barcelona. En: Libro de proceedings, CTV 2018. XII Congreso Internacional Ciudad y Territorio Virtual. "Ciudades y Territorios Inteligentes". UNCuyo, Mendoza, 5-7 septiembre 2018. Barcelona: CPSV, 2018, p. 339-356. 
Tabla 3. Unidades de consumo energético y emisiones preferidas

Máximo nivel de estudios

Toda la muestra Pregrado Grado Postgrado
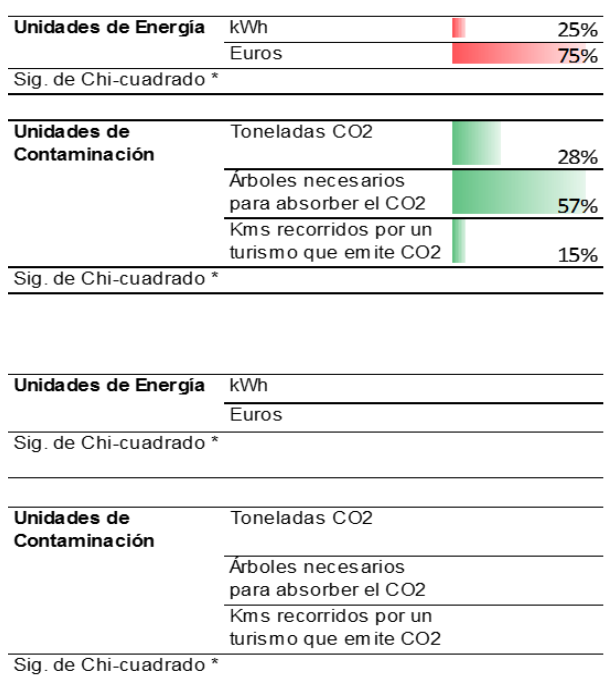
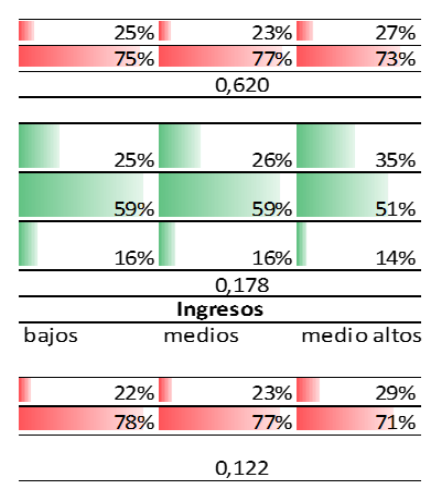

$$
0,122
$$

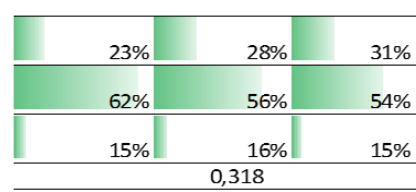

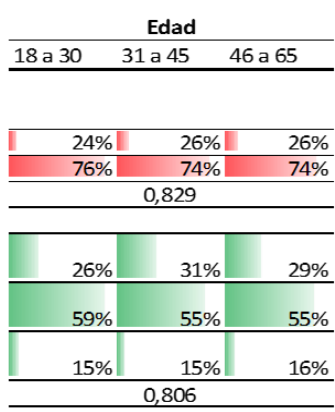

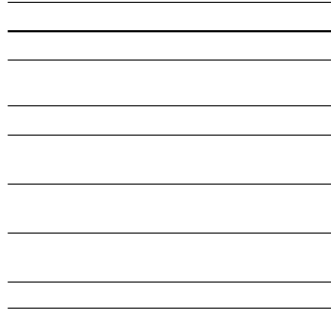

Fuente: Elaboración propia.

Si se realiza el análisis segmentado se aprecia que, en el mayor nivel de estudios "postgrado" se prefieren las unidades de masa, en un mayor porcentaje que en los niveles inferiores, aunque solo en un $35 \%$, similar al $31 \%$ que las prefiere en el caso de los ingresos "medios altos". En ambos caos, muy próximos a un tercio de la muestra. Se debe destacar que las unidades en árboles equivalentes son con diferencia las mayoritariamente preferidas, en todos los segmentos, de cualquiera de las clasificaciones de nivel de estudios e ingresos o grupos etarios, estando en todos los casos por encima del $50 \%$ del total.

\subsection{Disponibilidad a pagar por la mejora de la clase energética}

La inmensa mayoría de los 750 encuestados, 686 personas (un 91,5\%) declaró estar DAP más por la mejora de la clase energética, mientras que solo 64 personas (el 8,5\% restante) rechazó pagar más. Por ello, la primera conclusión es que, la gran mayoría de la población encuestada está DAP por acceder a viviendas energéticamente más eficientes.

Tabla 4. Razones de la no disposición a pagar

\begin{tabular}{lrc}
\hline Motivo & Frecuencia & Frecuencia relativa \\
\hline No le importa la eficiencia energética & 2 & $3 \%$ \\
\hline Ya paga demasiado por la vivienda & 23 & $36 \%$ \\
\hline El ahorro en la factura de luz y gas es insuficiente & 16 & $25 \%$ \\
\hline Su economía no le permite pagar más & 23 & $36 \%$ \\
\hline Total & 64 & $100 \%$ \\
\hline
\end{tabular}

Fuente: Elaboración propia.

Es importante indicar, como muestra en la Tabla 4, que 46 de las 64 personas que no estaban DAP (el $72 \%$ ), argumentaron cuestiones económicas; 23 personas (un 36\%) indicaron que ya pagaban demasiado por la vivienda y otras 23 que su economía no les permitía pagar más. Asimismo, solo 2 personas (un 3\%) declararon que no les importaba la eficiencia energética.

Citación: MARMOLEJO, C. et al. Disponibilidad a pagar por la eficiencia energética de las viviendas; un caso de estudio en Barcelona. En: Libro de proceedings, CTV 2018. XII Congreso Internacional Ciudad y Territorio Virtual. "Ciudades y Territorios Inteligentes". UNCuyo, Mendoza, 5-7 septiembre 2018. Barcelona: CPSV, 2018, p. 339-356. 
Para determinar la DAP media es necesario determinar las no-DAP que son ceros verdaderos y los que son ceros de protesta (Marmolejo et al, 2017). Para ello, sólo las 2 personas que indicaron que no pagaban por no importarles la eficiencia energética y las 16 consideraron que el ahorro era insuficiente fueron considerados ceros "verdaderos".

Tabla 5. Disposición a pagar según régimen de tenencia, nivel de estudios y de ingresos

\begin{tabular}{|c|c|c|c|c|c|}
\hline & & \multicolumn{2}{|c|}{ DAP } & \multirow[b]{2}{*}{$\begin{array}{l}\text { Ratio de } \\
\text { variación }\end{array}$} & \multirow[b]{2}{*}{$\begin{array}{l}\text { Sig. Test de } \\
\text { Anova }\end{array}$} \\
\hline & & $\begin{array}{c}\text { Media } \\
\text { (Eruos/mes) }\end{array}$ & $\begin{array}{l}\text { Desviación } \\
\text { estándar } \\
\text { (Euros/mes) }\end{array}$ & & \\
\hline \multirow{2}{*}{$\begin{array}{l}\text { Régimen de } \\
\text { tenencia deseado }\end{array}$} & Alquiler & 32,00 & 17,57 & 0,55 & \multirow{2}{*}{ 189 } \\
\hline & Propiedad & 35,00 & 22,29 & 0,64 & \\
\hline \multirow{3}{*}{ Nivel de estudios } & Pregrado & 33,70 & 21,37 & 0,63 & \multirow{3}{*}{ 740 } \\
\hline & Grado & 34,11 & 21,19 & 0,62 & \\
\hline & Postgrado & 32,70 & 17,77 & 0,54 & \\
\hline \multirow{3}{*}{ Nivel de ingresos } & Medio-bajo & 29,98 & 17,66 & 0,59 & \multirow{3}{*}{0,000} \\
\hline & Medio-alto & 32,90 & 19,12 & 0,58 & \\
\hline & Alto & 37,58 & 23,25 & 0,62 & \\
\hline
\end{tabular}

Fuente: Elaboración propia.

De media, las personas manifiestan una Disponibilidad a pagar de 33,70 euros/mes, por la mejora energética ofrecida. Sin embargo, este valor presenta variaciones importantes en la muestra segmentada. La Tabla 5 muestra que, según el régimen de tenencia deseado, quienes prefieren comprar están ligeramente más dispuesto a pagar que quienes prefieren alquilar, con valores de 35 y 32 euros/mes, respectivamente. Por su parte, el nivel de ingresos sí que condiciona la DAP; a más ingresos, mayor es la DAP, existiendo una diferencia de 7,60 euros/mes entre los 29,98 euros/mes de disponibilidad a pagar de las personas con nivel de ingresos "medio-bajo" y los 37,58 euros/mes de las personas con nivel de ingresos "alto". Mientras, que el nivel de estudios no presenta diferencias, ni tendencias significativas, aunque se debe indicar que son los que tienen grado, los que presentan la media más baja; 32,70 euros/mes.

\subsection{Razones que influencian la disponibilidad a pagar}

De las 686 personas que estaban DAP, 476 y 182 indicaron, respectivamente, que estarían "muy de acuerdo" y "de acuerdo" en hacerlo porque ahorrarían en la factura energética, mientras que, 394 y 234 indicaron, respectivamente, que estarían "muy de acuerdo" y "de acuerdo" en hacerlo porque contaminarían menos. Es muy relevante que 658 personas dieran importancia al ahorro en la factura energética, ya que se trata de una cuestión económica, pero también es relevante que una cifra muy similar; 628 personas dieran importancia al hecho de contaminar menos. De ello podemos deducir que, si bien el aspecto económico es relevante, no marca una diferencia preponderante. Esta situación también queda de manifiesto en las medias de valor obtenidas por las seis razones de la DAP, ${ }^{6}$ que en orden de importancia han sido: a) ahorro en la factura energética, con un 3,6; b) reducción de la contaminación, con un 3,$5 ;$ c) mejora en el confort y mejora en la salud, ambas con un 3,3; d) revalorización de la vivienda, con un 2,8 y e) por ser una acción socialmente ejemplar, con un 2,7.

${ }^{6}$ Calculadas con los siguientes valores: nada de acuerdo, 1 ; poco de acuerdo, 2; de acuerdo, 3 y muy de acuerdo; 4.

Citación: MARMOLEJO, C. et al. Disponibilidad a pagar por la eficiencia energética de las viviendas; un caso de estudio en Barcelona. En: Libro de proceedings, CTV 2018. XII Congreso Internacional Ciudad y Territorio Virtual. "Ciudades Y Territorios Inteligentes". UNCuyo, Mendoza, 5-7 septiembre 2018. Barcelona: CPSV, 2018, p. 339-356. 
Tabla 6. Motivos de la disposición a pagar, según segmentación de perfiles

\begin{tabular}{|c|c|c|c|c|c|c|c|c|c|}
\hline & \multirow[t]{2}{*}{ Total } & \multicolumn{3}{|c|}{ Nivel de estudios } & \multicolumn{5}{|l|}{ Edad } \\
\hline & & Pregrado & Grado & Postgrado & 18 a 30 & 31 a 45 & 46 a 65 & Más de 65 & 5 Sig Anova \\
\hline & Media & & & & & & & & \\
\hline Por los ahorros en la factura energética & 3,6268 & 3,6 & 3,7 & 3,6 & 3,7 & 3,6 & 3,6 & 3,5 & 0,881 \\
\hline Por contaminar menos & 3,4545 & 3,4 & 3,4 & 3,5 & 3,5 & 3,5 & 3,3 & 3,3 & 0,282 \\
\hline Porque es una acción socialmente ejemplar & 2,6268 & 2,8 & 2,5 & 2,7 & 2,6 & 2,7 & 2,6 & 2,6 & 0,85 \\
\hline Porque una viv energética vale más en el mercado & 2,8278 & 3,0 & 2,8 & 2,7 & 2,7 & 2,8 & 3,0 & 3,0 & 0,277 \\
\hline \multirow[t]{3}{*}{ Porque la salud mejora en un ambiente limpio } & 3,4354 & 3,5 & 3,5 & 3,4 & 3,4 & 3,4 & 3,5 & 3,2 & 0,379 \\
\hline & & \multicolumn{3}{|c|}{ Nivel de Ingresos } & & & & & \\
\hline & & M. bajos & M. altos & Altos & & & & & \\
\hline Por los ahorros en la factura energética & & 3,7 & 3,6 & 3,6 & & & & & \\
\hline Por contaminar menos & & 3,4 & 3,3 & 3,6 & & & & & \\
\hline Porque es una acción socialmente ejemplar & & 2,6 & 2,4 & 2,8 & & & & & \\
\hline Porque una viv energética vale más en el mercado & & 2,9 & 2,8 & 2,8 & & & & & \\
\hline Porque la salud mejora en un ambiente limpio & & 3,4 & 3,3 & 3,6 & & & & & \\
\hline
\end{tabular}

Tabla 7. Estadísticos descriptivos de las variables explicativas del modelo y su correlación con la DAP

\begin{tabular}{|c|c|c|c|c|c|c|c|c|}
\hline & Variable explicativa & $\begin{array}{l}\text { Correlación } \\
\text { de } \\
\text { Spearman } \\
\text { con el Ln de } \\
\text { la DAP }\end{array}$ & Sig. & Mín. & Máx. & Med & $\begin{array}{c}\text { Desviación } \\
\text { estandar }\end{array}$ & $\begin{array}{l}\text { Significado/ } \\
\text { unidades }\end{array}$ \\
\hline & Separa basura & 0,05 & 0,20 & 1 & 4 & 3,37 & 0,95 & 1= nunca, $4=$ siempre \\
\hline \multirow{2}{*}{$\begin{array}{c}\text { Comportamientos } \\
\text { sostenibles }\end{array}$} & Usa transporte público & 0,07 & 0,07 & 1 & 4 & 3,18 & 0,96 & $1=$ nunca, $4=$ siempre \\
\hline & Ahorra energía & 0,00 & 0,99 & 1 & 4 & 3,67 & 0,59 & 1=nunca, 4= siempre \\
\hline \multirow{5}{*}{$\begin{array}{l}\text { Prioridad para } \\
\text { mejorar la } \\
\text { vivienda actual }\end{array}$} & Reforma cocina y baños & 0,00 & 0,96 & 1 & 4 & 2,74 & 1,00 & 1=baja, 4=alta \\
\hline & Cambiar distribución espacios & 0,06 & 0,40 & 1 & 4 & 2,06 & 0,94 & 1=baja, 4=alta \\
\hline & Aislar muros & 0,13 & 0,06 & 1 & 4 & 2,75 & 1,04 & 1=baja, 4=alta \\
\hline & Aislar ventanas & 0,06 & 0,37 & 1 & 4 & 3,26 & 0,96 & 1=baja, 4=alta \\
\hline & Mejorar caldera & 0,08 & 0,26 & 1 & 4 & 2,86 & 0,99 & 1=baja, 4=alta \\
\hline \multirow{7}{*}{$\begin{array}{l}\text { Razones por las } \\
\text { que sí está DAP } \\
\text { más por la } \\
\text { vivienda mejor } \\
\text { calificada }\end{array}$} & Porlos ahorros en la factura & & & & & & & \\
\hline & energética & 0,04 & 0,27 & 1 & 4 & 3,63 & 0,58 & 1=nada $\mid$ 4= muy de acuerdo \\
\hline & Por contaminar menos & 0,03 & 0,38 & 1 & 4 & 3,45 & 0,70 & 1=nada | 4= muy de acuerdo \\
\hline & $\begin{array}{l}\text { Porque es una acción } \\
\text { socialmente ejemplar }\end{array}$ & 0,01 & 0,90 & 1 & 4 & 2,63 & 0,93 & 1=nada $\mid$ 4= muy de acuerdo \\
\hline & Porque una viv energética & & & & & & & \\
\hline & vale más en el mercado & 0,01 & 0,80 & 1 & 4 & 2,83 & 0,80 & 1=nada $\mid$ 4= muy de acuerdo \\
\hline & $\begin{array}{l}\text { Porque la salud mejora en un } \\
\text { ambiente limpio }\end{array}$ & 0,00 & 0,92 & 2 & 4 & 3,44 & 0,64 & 1=nada $\mid$ 4= muy de acuerdo \\
\hline \multirow[t]{13}{*}{ Otros indicadores } & $\begin{array}{l}\text { Eficiencia energética percibida } \\
\text { de la viv. actual }\end{array}$ & 0,07 & 0,08 & 1 & 3 & 2,12 & 0,65 & 1=baja, $3=a l t a$ \\
\hline & Estudios terminados & 0,01 & 0,81 & 1 & 7 & 5,38 & 1,34 & 1=primaria, 7=postgrado \\
\hline & Edad & 0,14 & - & 24 & 70 & 35,64 & 14,32 & años \\
\hline & Ingreso neto familiar & 0,13 & 0,00 & 600 & 4800 & 2.618 & 1.328 & Euros/mes \\
\hline & Conocimiento_EPC & 0,06 & 0,22 & 0 & 4 & 2,51 & 0,88 & $0=$ nulo, $4=$ muy alto \\
\hline & Conocimiento_EPC con EVP3 & 0,01 & 0,85 & 0 & 4 & 3,01 & 1,00 & \\
\hline & EVP1 & 0,04 & 0,27 & 0 & 1 & 0,33 & 0,47 & \\
\hline & EVP2 & 0,00 & 0,93 & 0 & 1 & 0,33 & 0,47 & \\
\hline & EVP3 & 0,05 & 0,24 & 0 & 1 & 0,33 & 0,47 & \\
\hline & Reforma_energ* & 0,01 & 0,79 & 3 & 12 & 8,87 & 2,28 & \\
\hline & Med_amb** & 0,00 & 0,93 & 3 & 12 & 10,22 & 1,59 & \\
\hline & Mujer & 0,11 & 0,01 & 0 & 1 & 0,48 & 0,50 & $(\%)$ \\
\hline & $\begin{array}{l}\text { Prefiere comprar la vivienda } \\
\text { ofrecida }\end{array}$ & 0,04 & 0,29 & 1 & 2 & 1,60 & 0,49 & $(\%)$ \\
\hline \multirow{4}{*}{$\begin{array}{l}\text { Relación con la } \\
\text { acividad }\end{array}$} & Estudiante & 0,08 & 0,03 & 0 & 1 & 0,35 & 0,48 & $(\%)$ \\
\hline & Desempleado/a & 0,02 & 0,64 & 0 & 1 & 0,03 & 0,17 & (\%) \\
\hline & Trabajando & 0,08 & 0,03 & 0 & 1 & 0,57 & 0,49 & $(\%)$ \\
\hline & Jubiado/a u otro & 0,01 & 0,79 & 0 & 1 & 0,04 & 0,20 & (\%) \\
\hline
\end{tabular}

Fuente: Elaboración propia. Notas: * variable sintética a partir de identificar las personas que indican como prioritario la mejora del aislamiento de los muros, las ventanas y la sustitución de las calderas por otras más eficientes de su vivienda actual, ${ }^{* *}$ variable sintética a partir de analizar los comportamientos sostenibles de los encuestados. En gris aparecen las variables cuya correlación con el Ln de la DAP no es significativa al $90 \%$ de confianza.

Citación: MARMOLEJO, C. et al. Disponibilidad a pagar por la eficiencia energética de las viviendas; un caso de estudio en Barcelona. En: Libro de proceedings, CTV 2018. XII Congreso Internacional Ciudad y Territorio Virtual. "Ciudades y Territorios Inteligentes". UNCuyo, Mendoza, 5-7 septiembre 2018. Barcelona: CPSV, 2018, p. 339-356. 
Si se realiza el análisis de los motivos de la disposición a pagar, según la segmentación (Tabla 6 ), se puede ver que no hay grandes variaciones internas entre los diferentes rangos de cada uno; nivel de estudios, nivel de ingresos o edad. Por ejemplo, en el caso del nivel de estudios, siempre la causa mayor es el "ahorro en la factura energética", con un 3,6 de media en los encuestados con pregrado, un 3,7 en los que tienen grado y un 3,6 en los de postgrado. Una situación similar es la que encontramos en la segmentación por edades, en que el "ahorro en la factura energética", también es la causa más relevante, con medias desde 3,7 a 3,5, desde los más jóvenes a los mayores. Por su parte, la misma lógica se repite en el nivel de ingresos, con medias de 3,6 y 3,7 en los tres segmentos. Cabe destacar también que el "contaminar menos" y las "mejoras de la salud en un ambiente limpio" se manifiestan como segunda y tercera causa, respectivamente, todos los segmentos, siempre con medias, entre el 3,3 y el 3,6. Por su parte el considerarlo una "acción socialmente ejemplar" siempre es la causa menos valorada.

Para identificar los factores que inciden en la DAP se realiza un análisis multivariado, mediante un modelo de regresión cuya variable dependiente es el Ln de la DAP (Marmolejo et al, 2017) y las independientes, las que se muestran en la Tabla 7. De ésta, cabe destacar la correlación positiva entre la DAP y "edad" e "ingreso neto familiar", reforzando los análisis previos. Los resultados del modelo de regresión (con limitaciones de ajuste, pero estadísticamente significativo), permiten determinar que el principal elemento explicativo de la DAP es el nivel de ingresos. Por cada 1.000 euros de incremento de le renta familiar disponible, el incremento de la DAP es prácticamente de un $4,5 \%$. A continuación, aparece "una mejora en la salud", en que por cada escalón que incrementa dicho indicador, la DAP incrementa un $8 \%$.

\section{Discusión y conclusiones}

Como se ha indicado previamente, la universalización de los (EPC) Certificados de Eficiencia Energética, con motivo de la venta y el alquiler de, prácticamente cualquier inmueble, deviene de la apuesta por la promoción de edificios eficientes, mediante el fomento de transacciones inmobiliarias energéticamente informadas, producto de la Directiva de Eficiencia Energética de Edificios (2002/91/EC) refundida en la EPBD 2010/31UE). Sin embargo, la tardía trasposición de la Directiva en España, mediante el RD 235/2013 y la posterior crisis económica (con la consecuente reducción de operaciones inmobiliarias) han ralentizado la universalización de los EPC en nuestro mercado inmobiliario. Esta falta de un volumen significativo de operaciones inmobiliarias (reales) para estudiar la incidencia de los EPC no permite estudiar la DAP de manera directa. Siendo así, en este trabajo se utiliza la Valoración Contingente (VC) basada en encuestas para evaluar: a) el nivel de conocimiento de los EPC por parte de los encuestados; b) si existe disposición a pagar por viviendas más eficientes energéticamente, y c) las razones subyacentes, en esta disposición a pagar (DAP) o de su rechazo. Por su parte, la hipótesis del plantea que, si se informa más claramente a la demanda, sobre las características e implicaciones de la certificación energética, se genera una mayor DAP, de los usuarios.

Los resultados, obtenidos indican que, en general, hay un bastante desconocimiento de lo que miden los EPC (sólo un 10\% de los encuestados tiene un conocimiento alto de ello), sobretodo en el segmento de población con un nivel educativo bajo y en las personas de mayor edad.

Sin embargo, a pesar de este desconocimiento, se determina una importante disposición a pagar por una vivienda energéticamente mejor. Un $91,5 \%$ de los encuestados declaró estar dispuesto a pagar más por la mejora de la clase energética, y además que, esta disposición

Citación: MARMOLEJO, C. et al. Disponibilidad a pagar por la eficiencia energética de las viviendas; un caso de estudio en Barcelona. En: Libro de proceedings, CTV 2018. XII Congreso Internacional Ciudad y Territorio Virtual. "Ciudades y Territorios Inteligentes". UNCuyo, Mendoza, 5-7 septiembre 2018. Barcelona: CPSV, 2018, p. 339-356. 
estaba justificada como razón principal, en gran medida, en el "ahorro en la factura energética". Por tanto, se puede concluir que efectivamente, si los usuarios reciben una mejor información acerca de las implicaciones de los EPC, la disposición a pagar aumenta. La DAP media es de 33,70 euros/mes, sin embargo, este valor presenta variaciones importantes en la muestra segmentada, mostrando que, quienes prefieren comprar están ligeramente más dispuesto a pagar que quienes prefieren alquilar, con valores de 35 y 32 euros/mes, respectivamente. Esto conlleva un importante desafío para las políticas de vivienda recientes, que han apostado por el alquiler, puesto que las personas encuestadas continúan entendiendo la compra como una inversión que merece un esfuerzo adicional, algo mayor que el caso del alquiler.

Por otra parte, el nivel de ingresos condiciona la DAP; a más ingresos, mayor es la DAP, existiendo una diferencia de 7,60 euros/mes entre los 29,98 euros/mes de disponibilidad a pagar de las personas con nivel de ingresos "medio-bajo" y los 37,58 euros/mes de las personas con nivel de ingresos "alto".

También se debe destacar que la gran mayoría de las personas (aproximadamente un 75\%) prefería recibir la información tanto de "unidades de consumo", como "unidades de contaminación", en "unidades ilustrativas, muy por encima de las que preferían las unidades técnicas, definidas por la regulación, que solo estaba alrededor del $25 \%$ de los encuestados. En cualquier caso, se debe especificar, que estas preferencias están condicionadas por el nivel de estudios, ya que, a mayor nivel formativo de las personas, tienen mejor aceptación las unidades técnicas ( $\mathrm{kWh}$ y toneladas). Todo esto se corresponde, en buena medida, con lo descubierto por Ramos et al. (2015) en el sentido que sólo, mediante una mejor información y educación a la población, se conseguirá el objetivo perseguido por la política energética europea.

Para finalizar, se puede indicar, que la disposición a pagar, no solo está condicionada por factores económicos, como el nivel de renta o los costes energéticos evitados, sino también sociales y, además, el nivel de conocimiento de los EPC y la preferencia del régimen en propiedad respecto del alquiler también inciden en las decisiones de pago. $\mathrm{Y}$, como síntesis, se destacan algunos de los aspectos relevantes presentados:

- El régimen de tenencia deseado mayoritariamente es la compra que, con 448 respuestas, alcanza el $59,7 \%$ del total de los encuestados.

- El desconocimiento generalizado del esquema certificatorio y de las implicaciones de los EPC, genera una infravaloración de las viviendas más eficientes, sin embargo, una mejora en la información y más claridad en las unidades de medida, mejora su DAP.

- A pesar del desconocimiento, la gran mayoría de los encuestados, 686 (equivalentes al $78 \%$ ) está dispuesto a realizar un pago adicional por una mejora en la eficiencia energética de la vivienda, mientras que sólo 64 no lo está.

- Quienes no están DAP argumentaron básicamente los motivos económicos; su economía no lo permite o ya paga demasiado por la factura (cada uno de ellos con un $36 \%$ )

- Por su parte, "el ahorro en la factura energética" se sitúa como principal razón de DAP, con una valoración media total de 3,68, al igual que en cada uno de los segmentos, situándose como primera razón en todos los casos, seguida por "contaminar menos" y "mejora de la salud en un ambiente limpio", con medias de 3,45 y 3,43 respectivamente, repartidas de manera muy similar en todos los segmentos estudiados.

Citación: MARMOLEJO, C. et al. Disponibilidad a pagar por la eficiencia energética de las viviendas; un caso de estudio en Barcelona. En: Libro de proceedings, CTV 2018. XII Congreso Internacional Ciudad y Territorio Virtual. "Ciudades y Territorios Inteligentes". UNCuyo, Mendoza, 5-7 septiembre 2018. Barcelona: CPSV, 2018, p. 339-356. 


\section{Agradecimientos}

Este trabajo ha sido desarrollado gracias al financiamiento recibido por el proyecto EnerValor ¿Cuánto nos importa la calificación energética? Un análisis del nivel de comprensión de los EPC, confianza percibida e impacto sobre las preferencias y valores residenciales (BIA201563606-R - MINECO/FEDER). Por ello se reconoce y agradece la aportación de las agencias financiadoras del proyecto, sin la que no se hubiese podido realizar.

\section{Bibliografía}

AZQUETA, D. Valoración económica del medio ambiente: una revisión crítica de los métodos y sus limitaciones. En: Información Comercial Española, 1996, 751: 37-46.

BARBOZA, M. Evaluación de costes y beneficios de una mayor cualificación energética en el mercado residencial de nueva planta en Barcelona. Tesis de máster. Universidad Politécnica de Cataluña, UPC. [En línea] 2016. Barcelona. Disponible en: http://hdl.handle.net/2117/84402

BROUNEN, D. y KOK, N. On the economics of energy labelling in the housing market. En: Journal of Environmental Economics and Management [en línea], 2011, 62 (2): 166-179. Fecha de consulta: 03 de julio de 2018. DOI: https://doi.org/10.1016/j.jeem.2010.11.006

CENTRE FOR HUMAN SETTLEMENTS (UN-HABITAT). City planning will determine pace of global warming. [En línea], 30 de octubre de 2007. Fecha de consulta: 03 de julio de 2018. Disponible en: http://www.un.org/press/en/2007/gaef3190.doc.htm

CHECA, C. y BIERE, R. Aproximación a la influencia de las certificaciones energéticas en los valores inmobiliarios. En: ACE: Architecture, City and Environment [en línea], octubre 2017, 12 (35): 165-190. Fecha de consulta: 3 de julio de 2018. Disponible en: http://hdl.handle.net/2117/109642 DOI: https://doi.org/10.5821/ace.12.35.5361

CE. Directiva del Parlamento Europeo y del Consejo relativa al rendimiento energético de los edificios (refundición). [En línea]. 2008. Fecha de consulta: 21 de junio de 2017. Disponible en: http://ec.europa.eu/transparency/regdoc/rep/1/2008/ES/1-2008-780-ES-F1-1.Pdf

DE AYALA, A.; GALARRAGA, I. y SPADARO, J. V. The price of energy efficiency in the Spanish housing market. En: Energy Policy [en línea], 2016, 94: 16-24. Fecha de consulta: 21 de junio de 2017. DOI: https://doi.org/10.1016/j.enpol.2016.03.032

DEL SAZ SALAZAR, S. \& SUÁREZ-BURGUET, C. El valor de uso recreativo de espacios naturales protegidos: aplicación del método de valoración contingente al Parque de l'Albufera. En: Revista española de economía agraria, 1998, №. 182, 239-271. Disponible en: https://www.mapa.gob.es/ministerio/pags/biblioteca/revistas/pdf reea/r182 07.pdf

EICHHOLTZ, P., NILS, K. y QUIGLEY, J. M. Doing Well by Doing Good? Green Office Buildings. En: American Economic Review [en línea] abril 2010, 100, 2492-2509. Disponible en: https://econpapers.repec.org/article/aeaaecrev/v 3a100 3ay 3a2010 3ai 3a5 3ap 3a2492-2509.htm

Citación: MARMOLEJO, C. et al. Disponibilidad a pagar por la eficiencia energética de las viviendas; un caso de estudio en Barcelona. En: Libro de proceedings, CTV 2018. XII Congreso Internacional Ciudad y Territorio Virtual. "Ciudades y Territorios Inteligentes". UNCuyo, Mendoza, 5-7 septiembre 2018. Barcelona: CPSV, 2018, p. 339-356. 
EPBD. Energy performance of buildings. [En línea] 2010. Fecha de consulta: 21 de junio 2017. Disponible en: https://ec.europa.eu/energy/sites/ener/files/documents/1 en act part1 v10.pdf

FUERST, F. y MCALLISTER, P. The impact of Energy Performance Certificates on the rental and capital values of commercial property assets. En: Energy Policy [en línea], octubre 2011, 39 (10): 6608-6614. Fecha de consulta: 1 de septiembre de 2018 . DOI: https://doi.org/10.1016/..enpol.2011.08.005

FUERST, F.; MCALLISTER, P.; NANDA, A. y WYATT, P. Does energy efficiency matter to home-buyers? An investigation of EPC ratings and transaction prices in England. En: Energy Economics [en línea], marzo 2015, 48, 145-156. Fecha de consultado 21 de junio de 2017. DOI: https://doi.org/10.1016/j.eneco.2014.12.012

GARCÍA-HOOGHUIS, A. y NEILA, F. Javier. Modelos de transposición de las Directivas 2002/91/CE y 2010/31/UE "Energy Performance Building Directive" en los Estados miembros de la UE. Consecuencias e implicaciones. En: Informes de la Construcción [en línea], 2013, 65 (531): 289-300. Fecha de consulta: 3 de julio de 2018. DOI: https://doi.org/10.3989/ic.12.017

GARCÍA-NAVARRO, J.; GONZÁLEZ-DÍAZ, M. J. y VALDIVIESO, M. Estudio Precost\&e: evaluación de los costes constructivos y consumos energéticos derivados de la calificación energética en un edificio de viviendas situado en Madrid. En: Informes de la Construcción [en línea], 2014, 66 (535): 1-10. Fecha de consulta: 3 de julio de 2018. DOI: http://dx.doi.org/10.3989/ic.13.052

HYLAND, M.; LYONS, R. y LYONS, S. The value of domestic building energy efficiency: evidence from Ireland. En: Energy Economics [en línea], noviembre 2013, 40, 943-952. Fecha de consulta: 3 de julio de 2018. DOI: https://doi.org/10.1016/j.eneco.2013.07.020

IPCC. Fourth Assessment Report: Climate Change 2007 (AR4). [En línea] Suiza. 2007. Fecha de consulta: 01 de septiembre de 2018. Disponible en: https://www.ipcc.ch/site/assets/uploads/2018/05/ar4 wg1 full report-1.pdf

KOK, N. y JENNEN, M. The impact of energy labels and accessibility on office rents. En: Energy Policy [En línea] Julio 2012, 46, 489-497. Fecha de consulta: 3 de junio de 2018. DOI: https://doi.org/10.1016/j.enpol.2012.04.015

LAINÉ, L. Room for improvement: The impact of EPCs on consumer decision-making [en línea]. London: Consumer Focus, 2011. Fecha de consulta: 01 de septiembre de 2018. Disponible en: http://www.epcmortgage.org.uk/assets/uploads/documents/ConsumerFocus Room-for-improvement.pdf

MARMOLEJO-DUARTE, C. La incidencia de la calificación energética sobre los valores residenciales: un análisis para el mercado plurifamiliar en Barcelona. En: Informes de la Construcción [en línea], 2016, 68 (543): e156. Fecha de consulta: 21 de junio de 2017. DOI: http://dx.doi.org/10.3989/ic.16.053

MARMOLEJO-DUARTE, C.; GARCÍA-HOOGHUIS, A. y GARCÍA-MASIÁ, A. ¿Cuánto nos importa la clase energética de nuestras viviendas? un análisis del nivel de comprensión de los EPC, disposición y motivos de pago en Barcelona. En: Revista Hábitat Sustentable. [En línea]. Junio 2017, 7 (1): 54-65. Fecha de consulta: 1 de septiembre de 2018. DOI: https://doi.org/10.22320/07190700.2017.07.01.06

Citación: MARMOLEJO, C. et al. Disponibilidad a pagar por la eficiencia energética de las viviendas; un caso de estudio en Barcelona. En: Libro de proceedings, CTV 2018. XII Congreso Internacional Ciudad y Territorio Virtual. "Ciudades y Territorios Inteligentes". UNCuyo, Mendoza, 5-7 septiembre 2018. Barcelona: CPSV, 2018, p. 339-356. 
MITCHELL, R. C. y CARSON, R. T. Using Surveys to Value Public Goods: The Contingent Valuation Method. Washington, D.C.: Resources for the Future, 1989.

MUDGAL, S.; LYONS, L. y COHEN, F. Energy performance certificates in buildings and their impact on transaction prices and rents in selected EU countries. Final report prepared for European Commission (DG Energy) [en línea], 2013. Fecha de consulta: 21 de junio de 2017. Disponible en: http://www.buildingrating.org/file/1566/download

POEL, B.; VAN CRUCHTEN, G. y BALARAS, C. Energy performance assessment of existing dwellings. En: Energy and Buildings. [En línea] 2007, 39 (4): 393-403. Fecha de consulta: 21 de junio de 2017. DOI: https://doi.org/10.1016/j.enbuild.2006.08.008

RAMOS, A.; GAGO, A.; LABANDEIRA, X. y LINARES, P. The role of information for energy efficiency in the residential sector. En: Energy Economics [en línea], 2015, 52, S1, S17-S29. Fecha de consulta: 1 de septiembre de 2018. DOI: https://doi.org/10.1016/i.eneco.2015.08.022

RIERA, P. Manual de Valoración Contingente. Madrid: Instituto de Estudios Fiscales, 1994.

ROMO, J. M.; MARMOLEJO, C. y DAUMAL, F. ¿Cuánto estamos dispuestos a pagar por vivir mejor insonorizados?: Un análisis para el mercado residencial de Barcelona. En: Revista de la construcción [en línea], 2013, 12 (2): 88-101. Fecha de consulta: 1 de septiembre de 2018. DOI: http://dx.doi.org/10.4067/S0718-915X2013000200007

SÁNCHEZ SÁEZ, J. y SEGADO SEGADO, F. y GIMÉNEZ MARTíNEZ, L. Valoración del uso recreativo en los Espacios Naturales Protegidos de la Región de Murcia. En: Cultura, Ciencia y Deporte [en línea] 2013, 8 (24): 223-230. Fecha de consulta: 08 de agosto de 2018. Disponible en: https://www.redalyc.org/articulo.oa?id=163028753007

TRENBERTH, K. E., et al. Estimates of the Global Water Budget and Its Annual Cycle Using Observational and Model Data. En: Journal of hydrometeorology. Special section [En línea]. Agosto 2007, 8: 758-769. Fecha de consulta: 08 de agosto de 2018. Disponible en: https://journals.ametsoc.org/doi/full/10.1175/JHM600.1 DOI: https://doi.org/10.1175/JHM600.1

WWF. Potencial de ahorro energético y de reducción de emisiones de $\mathrm{CO} 2$ del parque residencial existente en España en 2020. [En línea] 2010. Fecha de consulta: 07 de junio 2018. Disponible en: http://awsassets.wwf.es/downloads/informe potencial rehab vf dic2010.pdf

Citación: MARMOLEJO, C. et al. Disponibilidad a pagar por la eficiencia energética de las viviendas; un caso de estudio en Barcelona. En: Libro de proceedings, CTV 2018. XII Congreso Internacional Ciudad y Territorio Virtual. "Ciudades y Territorios Inteligentes". UNCuyo, Mendoza, 5-7 septiembre 2018. Barcelona: CPSV, 2018, p. 339-356. 